\title{
IMPULSIVE CONTROL OF RUMOURS WITH TWO BROADCASTS
}

\author{
SELMA BELEN' ${ }^{1}$, C. YALÇIN KAYA ${ }^{2}$ and C. E. M. PEARCE ${ }^{3}$
}

(Received 1 November, 2003; revised 5 November, 2004)

\begin{abstract}
In this paper we introduce an impulsive control model of a rumour process. The spreaders are classified as subscriber spreaders, who receive an initial broadcast of a rumour and start spreading it, and nonsubscriber spreaders who change from being an ignorant to being a spreader after encountering a spreader. There are two consecutive broadcasts. The first starts the rumour process. The objective is to time the second broadcast so that the final proportion of ignorants is minimised. The second broadcast reactivates as spreaders either the subscriber stiflers (Scenario 1) or all individuals who have been spreaders (Scenario 2). It is shown that with either scenario the optimal time for the second broadcast is always when the proportion of spreaders drops to zero.
\end{abstract}

\section{Introduction}

In a companion article [2], Belen and Pearce analysed the evolution from general initial conditions of the (common) deterministic limiting version of the Daley-Kendall and Maki-Thompson stochastic rumour models. Rumour models can be used to describe a variety of phenomena, such as the dissemination of information, disinformation or memes and changes in political persuasion and the stock market, for some of which the standard assumption of a single initial spreader and no initial stiflers is inappropriate. Results for a rumour process with general initial conditions are also relevant for the present study, which envisages a second rumour process developing in a situation created by a first.

In an age of mass communication, it is natural to consider the initiation of a rumour by means of television, radio or the internet (Frost [4]). We use the generic term

\footnotetext{
'School of Mathematics, The University of Adelaide, Adelaide SA 5005, Australia; e-mail: sbelen@ankara.baskent.edu.tr.

${ }^{2}$ School of Mathematics and Statistics, University of South Australia, Mawson Lakes SA 5095 ,

Australia; e-mail: yalcin.kaya@unisa.edu.au.

${ }^{3}$ School of Mathematics, The University of Adelaide, Adelaide SA 5005, Australia; e-mail:

cpearce@maths.adelaide.edu.au.

(C) Australian Mathematical Society 2005, Serial-fee code 1446-1811/05
} 
broadcast to refer to such an initiation. This paper envisages a control ingredient being added to a rumour model by the introduction of one or more subsequent broadcasts, with the intention of reducing the final proportion of the population never hearing the rumour. The rumour process is started by a broadcast to subscribers, who constitute the initial spreaders. Those ignorants who become spreaders after an encounter with a spreader we term nonsubscriber spreaders. We wish to determine, for given initial proportions of ignorants, spreaders and stiflers in the population, when to effect a second broadcast so as to minimise the final proportion of ignorants.

Two basic scenarios are considered. In the first, the recipients of the second broadcast are again the subscribers: a subscriber who had become a stifler is reactivated as a subscriber spreader. In Scenario 2, the recipients of the second broadcast are all individuals who were once spreaders.

As in [2], we describe the process in the continuum limit corresponding to a total population size tending to infinity. The resultant differential equations with each scenario can be expressed in state-space form, with the upward jump in subscriber spreaders modelled by an impulsive control input. Since we are dealing with an optimal control problem, a natural approach would be to employ a Pontryagin-like maximum principle furnishing necessary conditions for an extremum of an impulsive control system (see, for example, Blaquière [3] and Rempala and Zapcyk [6]). However, because of the tractability of the dynamical system equations, we are able to solve the given impulsive control problem without resorting to this theory.

The distinction between subscriber and nonsubscriber spreaders is of some independent interest. Before the second broadcast, these groups may be identified with two types of spreaders in an ordinary rumour, those who were spreaders initially and those who began as ignorants but became spreaders from an encounter with a spreader.

In Section 2 we refine the standard rumour model, studying the evolution of the proportions of these two groups in the population. In Section 3 we formulate Scenario 1 and derive associated results. We prove that the optimal time for the second broadcast is at the end of the first process, that is, when the proportion of spreaders in the population drops to zero. Section 4 parallels Section 3 for Scenario 2.

The development utilises the refinement of the basic rumour presented in the following section. An alternative approach that does not use these ideas is given elsewhere and used to consider multiple broadcasts [5].

\section{Refinement of the rumour model}

In this section we consider the evolution of the proportions of subscriber and nonsubscriber spreaders in a standard rumour model.

The Daley-Kendall model considers a population of $n$ individuals with subpopula- 
tions of ignorants, spreaders and stiflers. Denote the respective sizes of these subpopulations by $i, s$ and $r$. We define in addition the respective numbers of subscriber and nonsubscriber stiflers by $s_{1}$ and $s_{2}$, so that $s=s_{1}+s_{2}$. There is homogeneous mixing of individuals. The interactions which result in changes of subpopulation in time $d \tau$, along with their associated probabilities, are as follows.

\begin{tabular}{clc}
\hline Interaction & \multicolumn{1}{c}{ Transition } & Probability \\
\hline$i \rightleftharpoons s$ & $\left(i, s_{1}, s_{2}, r\right) \mapsto\left(i-1, s_{1}, s_{2}+1, r\right)$ & $i s d \tau+o(d \tau)$ \\
$s_{1} \rightleftharpoons s_{1}$ & $\left(i, s_{1}, s_{2}, r\right) \mapsto\left(i, s_{1}-2, s_{2}, r+2\right)$ & $s_{1}\left(s_{1}-1\right) / 2 d \tau+o(d \tau)$ \\
$s_{2} \rightleftharpoons s_{2}$ & $\left(i, s_{1}, s_{2}, r\right) \mapsto\left(i, s_{1}, s_{2}-2, r+2\right)$ & $s_{2}\left(s_{2}-1\right) / 2 d \tau+o(d \tau)$ \\
$s_{1} \rightleftharpoons s_{2}$ & $\left(i, s_{1}, s_{2}, r\right) \mapsto\left(i, s_{1}-1, s_{2}-1, r+2\right)$ & $s_{1} s_{2} d \tau+o(d \tau)$ \\
$s_{1} \rightleftharpoons r$ & $\left(i, s_{1}, s_{2}, r\right) \mapsto\left(i, s_{1}-1, s_{2}, r+1\right)$ & $s_{1} r d \tau+o(d \tau)$ \\
$s_{2} \rightleftharpoons r$ & $\left(i, s_{1}, s_{2}, r\right) \mapsto\left(i, s_{1}, s_{2}-1, r+1\right)$ & $s_{2} r d \tau+o(d \tau)$ \\
\hline
\end{tabular}

We now adopt a continuum formulation appropriate for $n \rightarrow \infty$. Let $i(\tau), s(\tau)(=$ $\left.s_{1}(\tau)+s_{2}(\tau)\right), r(\tau)$ denote respectively the proportions of ignorants, spreaders and stiflers in the population at time $\tau \geq 0$. The evolution of the limiting form of the model is prescribed by the deterministic dynamic equations

$$
\begin{aligned}
& \frac{d i}{d \tau}=-i s, \\
& \frac{d s_{1}}{d \tau}=-s_{1}(1-i), \\
& \frac{d s_{2}}{d \tau}=-s_{2}(1-2 i)+s_{1} i, \\
& \frac{d r}{d \tau}=s(1-i)
\end{aligned}
$$

with initial conditions

$$
i(0)=\alpha>0, \quad s_{1}(0)=\beta>0, \quad s_{2}(0)=0 \quad \text { and } \quad r(0)=\gamma \geq 0
$$

satisfying

$$
\alpha+\beta+\gamma=1 .
$$

We remark that (2.2) and (2.3) may be combined to provide

$$
\frac{d s}{d \tau}=-s(1-2 i)
$$

It is convenient to introduce the parameter $\theta=\theta(\tau):=i / \alpha$, the ratio of the proportion of ignorants at time $\tau$ to the initial proportion. In [2] the following dynamics and asymptotics were established for the basic continuum rumour process. 
THEOREM 2.1. The evolution of the rumour process prescribed by (2.1), (2.7) and (2.4) subject to (2.5) and (2.6) is given parametrically in terms of $i$ by

$$
\begin{aligned}
s & =\beta-2(i-\alpha)+\ln (i / \alpha) \\
& =\beta-2 \alpha(\theta-1)+\ln \theta
\end{aligned}
$$

and $r=1-i-s$.

The process evolves towards an asymptotic state $\left(i_{\infty}, 0, r_{\infty}\right)$, with

$$
i \downarrow i_{\infty}=i_{\infty}(\alpha, \beta) \text { as } \tau \rightarrow \infty
$$

and

$$
0<i_{\infty}<1 / 2 .
$$

The parameter $\theta_{\infty}:=i_{\infty} / \alpha$ satisfies the transcendental equation

$$
\theta_{\infty} e^{2 \alpha\left(1-\theta_{\infty}\right)}=e^{-\beta}
$$

Further, $s \rightarrow 0$ and $r(\tau) \uparrow r_{\infty}=1-i_{\infty}$ as $\tau \rightarrow \infty$

Where we wish to indicate that $s$ is regarded as a function of $i$ (defined by (2.8)), we put

$$
s=S(i):=\beta-2(i-\alpha)+\ln (i / \alpha) .
$$

The dynamics of the process are specified by augmenting Theorem 2.1 with the following result.

THEOREM 2.2. In the rumour control model prescribed by (2.1)-(2.4) with initial conditions (2.5), (2.6) and

$$
s_{1}(0)=\beta, \quad s_{2}(0)=0,
$$

we have

$$
s_{1}=s \exp \left(-\int_{i}^{\alpha} \frac{d u}{S(u)}\right)
$$

and

$$
s_{2}=s\left[1-\exp \left(-\int_{i}^{\alpha} \frac{d u}{S(u)}\right)\right] .
$$

We have that $s_{1}$ is strictly decreasing, with the asymptotics of $s_{1}$ and $s_{2}$ given by

(i) $\lim _{i \rightarrow i_{\infty}}\left(s_{1} / s\right)=0$,

(ii) $\lim _{1 \rightarrow i_{\infty}}\left(s_{2} / s\right)=1$. 
PROOF. Equations (2.1) and (2.2) combine to yield

or

$$
\frac{1}{s_{1}} \frac{d s_{1}}{d i}=\frac{1}{i s}(1-i)
$$

$$
d\left(\ln s_{1}\right)=\frac{1}{i s}(1-2 i) d i+\frac{1}{s} d i .
$$

Also we have on combining (2.1) and (2.7) that

$$
\frac{d s}{d i}=\frac{1-2 i}{i}
$$

from which we obtain $d\left(\ln s_{1}\right)=(1 / s) d s+(1 / s) d i$. Equation (2.13) now follows on integration and use of the initial condition (2.12). Equation (2.14) now follows from $s_{2}=s-s_{1}$.

That $s_{1}$ is strictly decreasing follows from (2.2). Because $s \rightarrow 0$ as $\tau \rightarrow \infty$, we have $s_{1} \rightarrow 0$ and $s_{2} \rightarrow 0$. By l'Hôpital's rule,

$$
\lim _{i \rightarrow i_{\infty}} \frac{s_{1}}{s}=\lim _{i \rightarrow i_{\infty}} \frac{d s_{1} / d i}{d s / d i}=\lim _{i \rightarrow i_{\infty}} \frac{s_{1}(1-i) /(i s)}{(1-2 i) / i}=\left(\lim _{i \rightarrow i_{\infty}} \frac{1-i}{1-2 i}\right) \lim _{i \rightarrow i_{\infty}} \frac{s_{1}}{s} .
$$

The last step follows from (2.10), which also gives (i). Part (ii) follows from $s=s_{1}+s_{2}$.

The asymptotics for $s_{1}$ and $s_{2}$ show that the spreader population changes in the course of time from consisting entirely of subscribers to consisting entirely of nonsubscribers.

Suppose that the second broadcast is made when $i=i_{b}$. Denote by $s_{1 b}$ and $s_{b}$ respectively the proportions of subscriber spreaders and all spreaders just before that broadcast. Also denote by $s_{1 b}^{+}$and $s_{b}^{+}$respectively the corresponding proportions immediately after the broadcast. Then by (2.13)

and by (2.8)

$$
s_{1 b}=s_{b} \exp \left[-\int_{t b}^{\alpha} \frac{d u}{S(u)}\right]
$$

$$
s_{b}=\beta-2\left(i_{b}-\alpha\right)+\ln \left(i_{b} / \alpha\right) .
$$

At any time after the second broadcast

$$
s=s_{b}^{+}-2\left(i-i_{b}\right)+\ln \left(i / i_{b}\right) .
$$




\section{Results for Scenario 1}

We now proceed to address Scenario 1 in which only the original subscribers become spreaders again. Under this scenario $s_{1 b}^{+}=\beta$ and so

$$
s_{b}^{+}=s_{1 b}^{+}+s_{b}-s_{1 b}=\beta+s_{b}\left[1-\exp \left(-\int_{i_{b}}^{\alpha} \frac{d u}{S(u)}\right)\right] .
$$

The rumour process stops when $s=0$. Let $i_{f}, i_{\infty}$ denote respectively the final proportion of ignorants in the population subsequent to the second broadcast and the proportion of ignorants at the end of a single rumour process. The problem of finding the optimum broadcast time, or equivalently the value $i=i_{b}$ when the second broadcast is made, can be posed as

$$
\min _{i_{b}} i_{f} \quad \text { subject to } s_{b}^{+}-2\left(i_{f}-i_{b}\right)+\ln \left(i_{f} / i_{b}\right)=0
$$

where

$$
\begin{aligned}
s_{b}^{+} & =\beta+s_{b}\left[1-\exp \left(-\int_{i_{b}}^{\alpha} \frac{d u}{S(u)}\right)\right] \text { and } \\
s_{b} & =\beta-2\left(i_{b}-\alpha\right)+\ln \left(i_{b} / \alpha\right) .
\end{aligned}
$$

TheOREM 3.1. For $0<\alpha \leq 1-\beta<1$, the minimum in Problem $\left(P_{1}\right)$ is given uniquely by $i_{b}=i_{\infty}=i_{\infty}(b)$.

PROOF. Implicit differentiation with respect to $i_{b}$ of the constraint equation in Problem $\left(P_{1}\right)$ provides

$$
\frac{\partial s_{b}^{+}}{\partial i_{b}}-2\left(\frac{\partial i_{f}}{\partial i_{b}}-1\right)+\frac{1}{i_{f}} \frac{\partial i_{f}}{\partial i_{b}}-\frac{1}{i_{b}}=0 .
$$

From (3.1) we have

$$
\frac{\partial s_{b}^{+}}{\partial i_{b}}=\frac{\partial s_{b}}{\partial i_{b}}-\left(\frac{\partial s_{b}}{\partial i_{b}}+1\right) \exp \left(-\int_{i_{b}}^{\alpha} \frac{d u}{S(u)}\right),
$$

so that by (2.16)

$$
\frac{\partial s_{b}^{+}}{\partial i_{b}}=\frac{\partial s_{b}}{\partial i_{b}}-\left(\frac{\partial s_{b}}{\partial i_{b}}+1\right) \frac{s_{1 b}}{s_{b}}
$$

Also from (2.17) we have

$$
\frac{\partial s_{b}}{\partial i_{b}}=\frac{1-2 i_{b}}{i_{b}}
$$


Elimination of $\partial s_{b} / \partial i_{b}$ and $\partial s_{b}^{+} / \partial i_{b}$ between (3.2)-(3.4) provides

$$
\frac{\partial i_{f}}{\partial i_{b}}=\frac{i_{f}}{1-2 i_{f}} \frac{1-i_{b}}{i_{b}} \frac{s_{1 b}}{s_{b}} .
$$

By Theorem 2.1 with initial state the state entered immediately after the second broadcast, we have $0<i_{f}<1 / 2$ and so $i_{f} /\left(1-2 i_{f}\right)>0$ and $i_{\infty}<i_{b}<\alpha$. The terms $\left(1-i_{b}\right) / i_{b}$ and $s_{1 b} / s_{b}$ are also positive, so $\partial i_{f} / \partial i_{b}>0$ for $i_{\infty}<i_{b}<\alpha$. All three quotients on the right in (3.5) are bounded above. By Theorem 2(i), $\partial i_{f} / \partial i_{b} \rightarrow 0$ as $i_{b} \rightarrow i_{\infty}$. Hence $i_{f}$ is minimised uniquely by the choice $i_{b}=i_{\infty}$. This completes the proof.

COROllaRY 3.2. Put $i_{\omega}:=\inf \left\{i_{f}: i_{b} \in\left[i_{\infty}, \alpha\right]\right\}$. If the second broadcast time is chosen to coincide with the first, the situation reduces to one of a single broadcast and $i_{f}$ becomes $i_{\infty}$. Since $i_{f}$ is strictly increasing as a function of $i_{b}$, we have

$$
i_{\omega} \leq i_{f} \leq i_{\infty} \quad \text { for } \quad i_{b} \in\left[i_{\infty}, \alpha\right]
$$

Generally, (2.9) gives

$$
\frac{i_{\infty}}{\alpha} \exp \left[2\left(\alpha-i_{\infty}\right)\right]=e^{-\beta}
$$

for the first broadcast and

$$
\frac{i_{\omega}}{i_{\infty}} \exp \left[2\left(i_{\infty}-i_{\omega}\right)\right]=e^{-\beta}
$$

for an optimal second broadcast. Multiplying these relations together provides

$$
\frac{i_{\omega}}{\alpha} \exp \left[2\left(\alpha-i_{\omega}\right)\right]=e^{-2 \beta} .
$$

If $\alpha+2 \beta \leq 1$, this relation has a physical interpretation: the final proportion of ignorants after two broadcasts, the second being optimally timed, is the same as that resulting from a single broadcast with initial conditions

$$
i(0)=\alpha \quad \text { and } \quad s(0)=2 \beta .
$$

Figure 1 presents (for $\alpha+\beta=1$ with six different values of $\beta$ ) graphs of the final proportion $i_{f}$ of ignorants after the second broadcast as a function of the proportion $i_{b}$ of ignorants at the time of the second broadcast.

COROLlaRY 3.3. Suppose $\alpha \rightarrow \alpha_{0} \neq 0$ and $\beta \rightarrow 0$. By (3.6), the limiting value of $i_{\omega}$ satisfies $\left(i_{\omega} / \alpha_{0}\right) e^{2\left(\alpha_{0}-i_{\omega}\right)}=1$ and so $i_{\omega}=i_{\infty}$. By Corollary $3.2, i_{f}=i_{\infty}$ for each choice of $i_{b} \in\left[i_{\infty}, \alpha\right]$, that is, the second broadcast is ineffective at any time. 


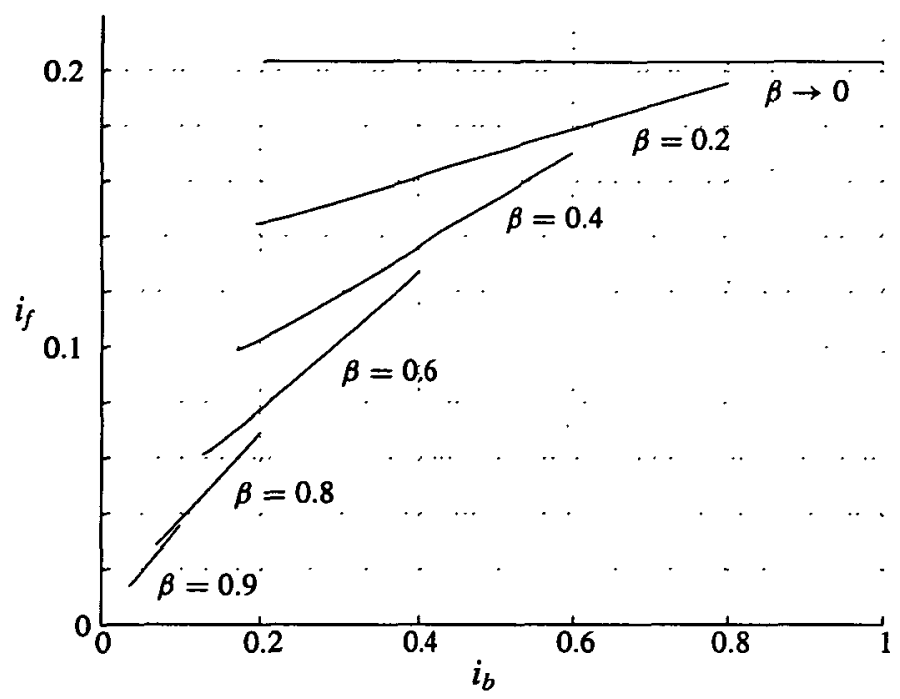

FIGURE $1 . i_{f}$ vs $i_{b}$ for various values of $\beta$ under Scenario 1 .

Intuitively this is not surprising. In the limiting case $\beta \rightarrow 0$, the reactivation of subscriber stiflers does not change the state, so $i_{f}$ has the same value for every $i_{b} \in\left[i_{\infty}, \alpha_{0}\right]$. In the case $\alpha \rightarrow 1$, the limit $i_{\infty} \approx 0.203$. We have accordingly the following result.

THEOREM 3.4. In the limiting case $\beta \rightarrow 0, \alpha \rightarrow \alpha_{0}>0$, any $i_{b} \in\left[i_{\infty}, \alpha_{0}\right]$ is $a$ solution of Problem $\left(P_{1}\right)$.

In the limit as $\alpha \rightarrow 0$ and $\beta \rightarrow \beta_{0} \neq 0$, we have from (2.11) that $\theta_{\infty}=e^{-\beta_{0}}$, so that for any feasible time for the second broadcast

$$
e^{-\beta_{0}}<\theta_{b} \leq 1 .
$$

LEMMA 3.5. Suppose $\alpha \rightarrow 0$ and $0<\beta_{0} \leq 1$. Then $\lim _{\beta \rightarrow \beta_{0}}\left(s_{1 b} / s_{b}\right)=1$.

PROOF. Since $i=\alpha \theta,(2.16)$ can be rewritten as

$$
\frac{s_{1 b}}{s_{b}}=\exp \left[-\alpha \int_{\theta_{b}}^{1}(1 / s) d \theta\right]
$$

so it suffices to show that $h:=\lim _{\alpha \rightarrow 0} \int_{\theta_{b}}^{1}(1 / s) d \theta<\infty$. From (2.9),

$$
h=\int_{\theta_{b}}^{1} \frac{d \theta}{\beta_{0}+\ln \theta}=e^{-\beta_{0}} \int_{e^{\beta_{0} \theta_{b}}}^{e^{\beta_{0}}} \frac{d u}{\ln u} .
$$




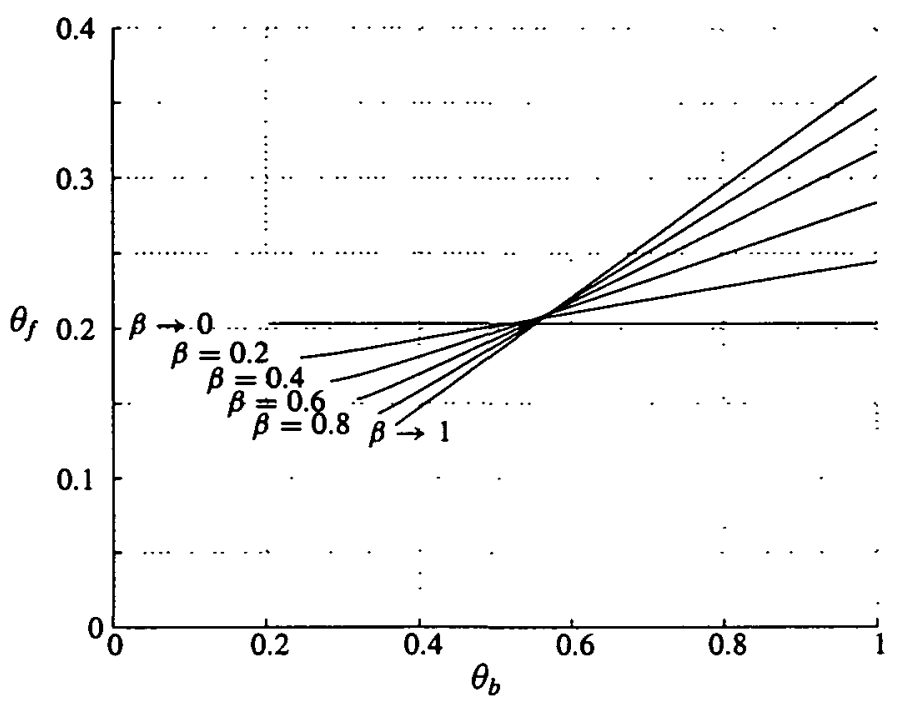

FIGURE 2. $\theta_{f}$ vs $\theta_{b}$ for various values of $\beta$ under Scenario 1 .

By Abramowitz and Stegun [1], Section 5.1, we may evaluate

$$
\int_{e^{\beta_{0} \theta_{b}}}^{e^{\beta_{0}}} \frac{d u}{\ln u}=E_{i}\left(\ln \left(e^{\beta_{0}}\right)\right)-E_{\imath}\left(\ln \left(e^{\beta_{0}} \theta_{b}\right)\right),
$$

where $E_{i}(x):=\gamma+\ln (\ln x)+\sum_{k=1}^{\infty}\left(\ln ^{k} x\right) /(k \cdot k !)$ and $\gamma$ is Euler's constant.

Hence (3.8) becomes

$$
h=e^{-\beta_{0}}\left[\ln \left(\frac{\beta_{0}}{\beta_{0}+\ln \left(\theta_{b}\right)}\right)+\sum_{k=1}^{\infty} \frac{\beta_{0}^{k}}{k \cdot k !}-\sum_{k=1}^{\infty} \frac{\ln ^{k}\left(e^{\beta_{0}} \theta_{b}\right)}{k \cdot k !}\right] .
$$

When $\theta_{b}=1, h=0$. On the other hand, when (3.7) holds with strict inequality, the leading term in brackets is finite. Both series in (3.9) converge absolutely. Hence $h$ is finite as required.

The result given by Lemma 3.5 provides an interesting contrast to Theorem 2.2 (i).

TheOREM 3.6. When $\alpha \rightarrow 0$ and $\beta \rightarrow \beta_{0} \neq 0$, Problem $\left(P_{1}\right)$ possesses the unique solution $\theta_{b}=\theta_{\infty}=e^{-\beta_{0}}$.

PROOF. With $i_{f}=\alpha \theta_{f}$ and $i_{b}=\alpha \theta_{b}$, (3.5) becomes

$$
\frac{\partial \theta_{f}}{\partial \theta_{b}}=\frac{\theta_{f}}{1-2 \alpha \theta_{f}} \frac{1-\alpha \theta_{b}}{\theta_{b}} \frac{s_{1 b}}{s_{\iota}} .
$$


Hence by Lemma 3.5, we have that in the limit

$$
\frac{\partial \theta_{f}}{\partial \theta_{b}}=\frac{\theta_{f}}{\theta_{b}},
$$

which is always positive. This completes the proof.

REMARK. We may deduce from (3.10) that $\partial^{2} \theta_{f} / \partial \theta_{b}^{2}=0$ in the limit $\beta \rightarrow \beta_{0}$, so that $\theta_{f}$ is linear in $\theta_{b}$. For $\alpha \rightarrow 0$ and $\beta \rightarrow \beta_{0}$, we have by Lemma 3.5 that the first constraint equation in Problem $\left(P_{1}\right)$ becomes $\beta_{0}+\ln \left(\theta_{f} / \theta_{b}\right)=0$. This yields $\theta_{f}=e^{-\beta_{0}} \theta_{b}$. As may be seen in Figure 2 for the special case $\alpha+\beta=1$, the slope of the graph of $\theta_{f}$ vs $\theta_{b}$ has the constant value $1 / e$ for $\beta \rightarrow 1$. The slope is not constant for $0<\beta<1$.

\section{Results for Scenario 2}

Under Scenario 2, we have $s_{b}^{+}=\alpha+\beta-i_{b}$ and so (2.18) becomes

$$
s=\alpha+\beta+i_{b}-2 i+\ln \left(i / i_{b}\right)
$$

for any time after the second broadcast.

The optimisation problem now has a much simpler form than in Problem $\left(P_{1}\right)$, namely

$$
\min _{i_{b}} i_{f} \quad \text { subject to } \alpha+\beta+i_{b}-2 i_{f}+\ln \left(i_{f} / i_{b}\right)=0
$$

When there are no stiflers initially in the population, that is, when $\alpha+\beta=1$, Problem $\left(P_{2}\right)$ is independent of $\beta$, in contrast to Problem $\left(P_{1}\right)$. Parallels to Theorems $3.1,3.4$ and 3.6 for this scenario can be combined into a single result, which we give below.

THEOREM 4.1. When $\alpha \rightarrow \alpha_{0}$ with $0<\alpha_{0} \leq 1, i_{b}=i_{\infty}$ is the unique solution to Problem $\left(P_{2}\right)$. When $\alpha \rightarrow 0$ and $\beta \rightarrow \beta_{0}$, with $0<\beta_{0} \leq 1, \theta_{b}=\theta_{\infty}=e^{-\beta_{0}}$ is the only solution.

Proof. First suppose $\alpha \rightarrow \alpha_{0}$ with $0<\alpha_{0} \leq 1$. Implicit differentiation of the constraint equation in Problem $\left(P_{2}\right)$ gives

$$
\left(\frac{1}{i_{f}}-2\right) \frac{\partial i_{f}}{\partial i_{b}}=\frac{1}{i_{b}}-1
$$




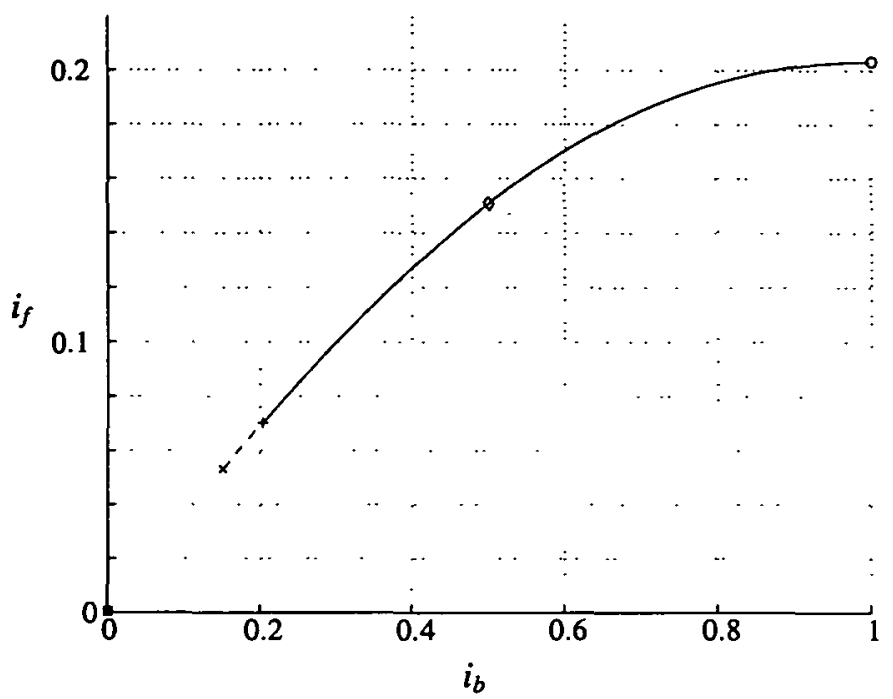

FIGURE 3. $i_{f}$ vs $i_{b}$ for various values of $\beta$ under Scenario 2. The curve segment startıng with $\circ$ and ending with + corresponds to the case $\beta \rightarrow 0$; the curve segment starting with $\diamond$ and ending with $\times$ corresponds to $\beta=0.5$. The case $\beta \rightarrow 1$ is given by a point at the origin.

or

$$
\frac{\partial i_{f}}{\partial i_{b}}=\frac{i_{f}}{1-2 i_{f}} \frac{1-i_{b}}{i_{b}} .
$$

Since $0<i_{f}<1 / 2$ and $0<i_{b}<1$, we have $\partial i_{f} / \partial i_{b}>0$, which implies, given $i_{\infty}<i_{b}<\alpha_{0}$, that $i_{b}=i_{\infty}$ is the unique solution.

Next consider $\alpha \rightarrow 0$ and $\beta \rightarrow \beta_{0}$, with $0<\beta_{0} \leq 1$, and let $i_{f}=\alpha \theta_{f}$ and $i_{b}=\alpha \theta_{b}$. Substitution into (4.2) gives

$$
\frac{\partial \theta_{f}}{\partial \theta_{b}}=\frac{\theta_{f}}{\theta_{b}}>0,
$$

which implies, given $e^{-\beta_{0}}<\theta_{b}<1$, that $\theta_{b}=\theta_{\infty}=e^{-\beta_{0}}$ is the only solution.

Figure 4 presents (for $\alpha+\beta=1$ with three different values of $\beta$ ) graphs of $i_{f}$ after the second broadcast as a function of $i_{b}$.

REMARK. As with Scenario 1, we have $\partial^{2} \theta_{f} / \partial \theta_{b}^{2}=0$ and through the constraint equation in Problem $\left(P_{2}\right)$ we obtain $\theta_{f}=e^{-\beta_{0}} \theta_{b}$. In the limit as $\beta \rightarrow 1$, the slope of the graph of $\theta_{f}$ vs $\theta_{b}$ has the constant value $1 / e$, as illustrated in Figure 3. 


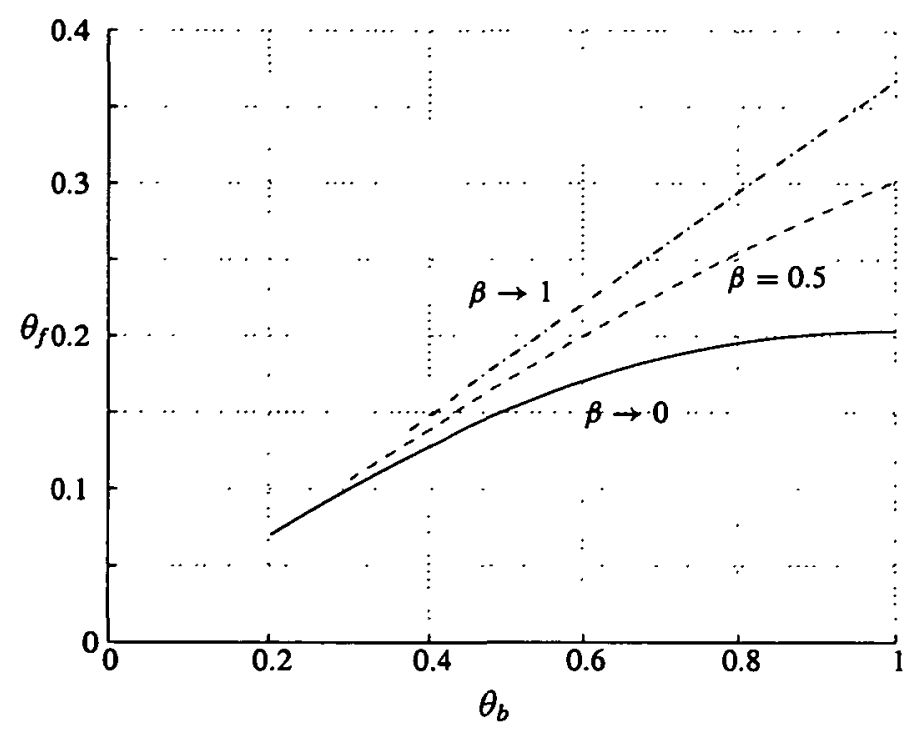

FIGURE 4. $\theta_{f}$ vs $\theta_{b}$ for various values of $\beta$ under Scenario 2 .

REMARK. Consider the case when there are no stiflers initially, that is, when $\alpha+\beta=1$. In Scenario 2, there are no stiflers left immediately after the second broadcast, all having become spreaders again. The larger the proportion of ignorants to have encountered the rumour before the second broadcast, the larger the proportion of stiflers present. So choosing a broadcast time with the highest proportion of stiflers (as in Theorem 4.1), that is, at the end of the process, is intuitively reasonable in order to achieve the lowest possible $i_{f}$.

The results for Scenario 1 are less obvious. Intuitively one might want the ratio $s / i$ to be as large as possible at the start of a process to increase the dispersal of the rumour. However a second broadcast at the end of the first process does not necessarily make $s / i$ larger than at earlier stages of the first process.

\section{Conclusions}

In this paper we have introduced an impulsive control model of a rumour process. We considered two consecutive broadcasts, the first one starting the rumour process. In both the cases when spreaders are reactivated from the subscriber stiflers (Scenario 1) as well as from those stiflers who once were spreaders (Scenario 2), we have shown that the optimal time for the second broadcast to minimise the final proportion of ignorants is always at the end of the process started by the first broadcast. In other words, a second rumour process commences once the first process terminates. 
Some of the auxiliary results we obtained are worth mentioning here, because of their practical significance. One result shows that the spreader population changes from consisting entirely of subscribers to consisting entirely of nonsubscribers at the termination of a process. This can perhaps be interpreted as to why the end of the first process is the best time for a second broadcast. Another result implies that the final proportion of ignorants after two broadcasts, the second being optimally timed, is the same as that resulting from a single broadcast with twice the initial proportion of spreaders, provided this is allowed by the physical constraints. If one considers the initial proportion of spreaders as the "resource" available to start the process, then it may be best to allocate as much of this resource as possible at the beginning of the process.

Allowing general initial conditions for the basic rumour process simplified proofs considerably in our analysis. It is natural to consider next an extension of this study to the case with a general number of broadcasts. The setting with general initial conditions and the results obtained in this paper form a basis for this extension.

\section{References}

[1] M. Abramowitz and I. A. Stegun, Handbook of mathematical functions (Dover, New York, 1965).

[2] S. Belen and C. E. M. Pearce, "Rumours with general initial conditions", ANZIAM J. 45 (2004) $393-400$.

[3] A. Blaquière, "Impulsive optimal control with finite or infinite time horizon", J. Optim. Theory Appl. 46 (1985) 431-439.

[4] C. Frost, "Tales on the internet: making it up as you go along", ASLIB Proc. 52 (2000) 5-10.

[5] C. E. M. Pearce, C. Y. Kaya and S. Belen, "Impulsive control of a sequence of rumour processes", submitted.

[6] R. Rempala and J. Zabczyk, "On the maximum principle for deterministic impulse control problems", J. Optim. Theory Appl. 59 (1988) 281-288. 